\section{Aceitabilidade da quimioprofilaxia em área endêmica para a hanseníase: projeto PEP-Hans Brasil}

\author{
Acceptability of chemoprophylaxis in an endemic \\ area for leprosy: the PEP-Hans Brazil Project
}

\section{Aceptabilidad de la quimioprofilaxis en un área endémica de hanseniasis: Proyecto PEP-Hans Brasil}

Denise da Costa Boamorte Cortela 1,2

Silvana Margarida Benevidez Ferreira 2,3

Marcos Cunha Lopes Virmond 4

Liesbeth Mieras 5

Peter Steinmann 6

Eliane Ignotti 1

Arielle Cavaliero 7

\section{Resumo}

O objetivo deste artigo foi analisar a aceitabilidade da quimioprofilaxia com rifampicina em dose única (PEP) entre os contatos, casos indices de hanseníase e profissionais da saúde e fatores relacionados que possam influenciar na adesão. Realizou-se um estudo qualitativo de análise de conteúdo após aplicação de entrevistas semiestruturadas segundo protocolo proposto no programa LPEP (2016), realizado em Alta Floresta, Mato Grosso, Brasil, em julho de 2016. Participaram do estudo indivíduos notificados com hanseniase, contatos e profissionais da saúde. Utilizou-se o software QRS NVivo versão 10. Foram contatados 80 indivíduos, sendo 54 (67\%) contatos, 11 (14\%) casos índices e 15 (19\%) profissionais de saúde. Dentre os contatos, 94\% (51/54) tomaram PEP. Foram identificadas 3 categorias quanto à PEP: compreensão, aceitação e expectativa da intervenção. A compreensão se mostrou relacionada ao cuidado da equipe de saúde. Aceitar ou não a medicação revelou-se relacionada ao medo, confiança e proteção, operacionalidade da estratégia, autoestima e insegurança quanto à intervenção. A expectativa da intervenção relacionou-se ao bem-estar, prevenção da doença e de sequelas, diminuição de gastos públicos e ampliação do acesso. Houve reconhecimento da relevância da estratégia PEP pela possibilidade de interrupção da cadeia de transmissão, diminuição de casos novos e melhora na qualidade de vida. A insegurança em tomar a medicação e de a doença se manifestar influenciaram negativamente à aceitação da PEP; por outro lado, as informações prévias sobre a estratégia PEP contribuíram para o fortalecimento da confiança nos profissionais de saúde e para a aceitabilidade da medicação.

Rifampicina; Quimioprofilaxia; Conhecimentos, Atitudes e Prática em Saúde; Aceitação pelo Paciente de Cuidados de Saúde
Correspondência

D. C. B. Cortella

Faculdade de Ciências da Saúde, Universidade do Estado de Mato Grosso.

Av. São João s/n, Campus Universitário Jane Vanini, Cáceres, MT 78200-000, Brasil.

denisecortela@hotmail.com

1 Faculdade de Ciências da Saúde, Universidade do Estado de Mato Grosso, Cáceres, Brasil.

2 Universidade Federal de Mato Grosso, Cuiabá, Brasil.

3 Universidade de Cuiabá, Cuiabá, Brasil.

4 Instituto Lauro de Souza Lima, Bauru, Brasil.

${ }^{5}$ Netherlands Leprosy Relief, Amsterdam, The Netherlands.

6 Swiss Tropical and Public Health Institute, Basel, Switzerland.

7 Novartis Foundation, Basel, Switzerland. 


\section{Introdução}

A hanseníase é uma doença incapacitante e deformante e, independentemente do sexo e da idade, pode comprometer adultos e crianças. Sabe-se que os contatos domiciliares, de vizinhança e sociais apresentam maior risco de adoecimento 1,2,3,4.

Para a vigilância da hanseníase, o exame de contatos é uma atividade estratégica e destacam-se como ações de controle o diagnóstico precoce, a regularidade terapêutica, os procedimentos reabilitacionais, as ações educativas e, recentemente, como estratégia adicional para redução da transmissão da hanseníase, a quimioprofilaxia em dose única de rifampicina oferecida aos contatos de casos conhecidos 5 .

A profilaxia pós-exposição tem sido o termo amplamente utilizado para a quimioprofilaxia e/ ou imunoprofilaxia da hanseníase 6 . As evidências apontam, após a administração de dose única de rifampicina, uma redução do risco de adoecimento entre contatos de $60 \%$ nos primeiros dois anos, sendo tal efeito mantido após 4 a 6 anos 7,8. Trata-se de uma estratégia socialmente aceita, mostrandose eficaz na prevenção da hanseníase entre contatos de indivíduos com hanseníase 9.

No Brasil, um programa piloto para a avaliação da operacionalidade da quimioprofilaxia com rifampicina em dose única (PEP) foi implementado na rotina de programas de hanseníase no âmbito do Sistema Único de Saúde (SUS), em Mato Grosso, Tocantins e Pernambuco e denominado projeto PEP-Hans Brasil 6.

A viabilidade e a implementação bem-sucedida do protocolo profilático PEP exige articulação em todos os níveis de atenção à saúde e algumas condições devem ser consideradas. Há necessidade de um planejamento operacional, de fatores técnicos, disponibilidades de recursos, conhecimento do estigma, da percepção, do impacto da distribuição da PEP e da aceitabilidade da intervenção 10,11 .

A aceitabilidade pode ser entendida como resultado de uma implementação relacionada à percepção, entre as pessoas participantes, de que um serviço, tratamento ou inovação é bom 11. No que tange aos cuidados ofertados pelos serviços de saúde, ela está vinculada à satisfação demonstrada por usuários e pela comunidade, à melhora na qualidade de vida e à adesão ao tratamento 12. Por outro lado, fatores relacionados diretamente ao indivíduo, isso é, sua condição sociodemográfica, cultura e a percepção sobre a doença podem comprometer a aceitabilidade e a adesão ao tratamento 13 .

Considerando a necessidade de maior conhecimento sobre a implementação da PEP e sua efetiva implantação no Brasil, este estudo objetiva a análise da aceitabilidade da PEP em um município endêmico no Estado de Mato Grosso, bem como os fatores relacionados que possam influenciar na adesão da intervenção.

\section{Metodologia e população}

Trata-se de um estudo qualitativo de análise da aceitabilidade da PEP entre indivíduos residentes no Município de Alta Floresta, Estado de Mato Grosso, realizado no segundo semestre de 2016.

Definiu-se como grupo-alvo para as entrevistas os casos índices de hanseníase, contatos intradomiciliares e de vizinhança, contatos sociais e profissionais da saúde. Entendeu-se por vizinho aquele indivíduo que morava na residência ao lado, na frente ou atrás da residência de um caso índice. Os contatos sociais corresponderam aos indivíduos que trabalhavam ou frequentavam alguma escola, igreja ou estabelecimento comercial localizado no território da unidade básica de saúde (UBS) de tratamento do caso índice e que não eram vizinhos ou contatos domiciliares do caso índice.

Considerou-se como critério de inclusão todo indivíduo com idade acima de 10 anos e residente na área adscrita da UBS visitada. Pessoas com déficit mental e incapacidade de responder às questões foram excluídas.

Para a coleta de dados, foram realizadas entrevistas semiestruturadas durante visitas domiciliares, em estabelecimentos comerciais, igrejas e em 10 unidades de saúde, segundo o método exposto no programa LPEP 14.

Informações sobre o gênero, idade, nível de escolaridade, crença e tipo de profissão foram coletadas por meio de questões semiabertas. 
Os encontros para as entrevistas foram previamente informados pelos agentes comunitários de saúde durante sua visita de rotina de trabalho nas residências do bairro da UBS. Quando o indivíduo se encontrava ausente, buscou-se agendar um novo momento para a entrevista e, após duas tentativas sem sucesso, ele foi excluído.

Todas as entrevistas foram gravadas em áudio e transcritas para a análise de conteúdo segundo Bardin 15 tendo em conta as fases: pré-análise; exploração do material e tratamento dos resultados; e inferência e interpretação. Utilizou-se o software QRS NVivo versão 10 (https://www.qsrinternatio nal.com/nvivo/home). Os dados referentes às informações sobre gênero, idade, nível de escolaridade, crença e tipo de profissão foram transferidas para uma planilha Microsoft Excel versão 2016 (https:// products.office.com/) para análise descritiva.

Para a análise da aceitabilidade da PEP, foram investigadas seis questões-chaves, listadas no Quadro 1. Durante as transcrições, procurou-se identificar palavras ou termos recorrentes que pudessem ser agrupados de acordo com as questões de investigação para posterior análise contextual.

Com a finalidade de se preservar a identidade dos entrevistados, foram utilizadas siglas seguidas de um número para a apresentação dos resultados e discussão. Assim, para citação do indivíduo caso índice (CI-1; CI-2 etc.); contato intradomiciliar (CTI-1; CTI-2 etc.); contato social (CS-1; CS-2 etc.); vizinho (V-1; V-2 etc.); profissional de saúde (PS-1; PS-2 etc.). Todos assinaram o Termo de Consentimento Livre e Esclarecido e Assentimento, quando menores de idade.

O estudo foi aprovado pelo Comitê de Ética em Pesquisa/Plataforma Brasil/Universidade do Estado de Mato Grosso, sob o parecer no 1.276.414, em 13 de outubro de 2015, CAAE:48652515.1.0000.5166.

\section{Resultados}

No estudo, foram contatados 80 indivíduos e, dentre os contatos entrevistados, 94\% (51/54) aceitaram tomar a PEP. Não aceitaram tomar a PEP dois jovens, contatos intradomiciliares de residências diferentes, e um adulto considerado vizinho de um caso índice.

Os grupos-alvo entrevistados compreenderam 13,8\% (11/80) de casos índices, 26,2\% (21/80) de contatos intradomiciliares, $27,5 \%(22 / 80)$ de contatos sociais, $13,8 \%(11 / 80)$ de vizinhos e $18,7 \%$ $(15 / 80)$ de profissionais de saúde. A média de idade entre os entrevistados foi de 43 anos, variando de 14 anos a 79 anos (DP: 15,15).

Verificou-se que 70\% (56/80) eram do gênero feminino, 61,3\% (49/80) haviam frequentado a escola até o Ensino Fundamental e 60\% (48/80) eram católicos. Quanto à profissão dos entrevistados, $30 \%(24 / 80)$ corresponderam a donas de casa ou aposentados, 19\% (15/80) autônomos e 15\% (12/80) eram enfermeiros ou agentes de saúde.

$\mathrm{Na}$ análise exploratória inicial durante consulta de frequência de palavras em todos os textos transcritos, identificou-se que os termos mais frequentes foram: hanseníase $(\mathrm{n}=328)$, informação $(\mathrm{n}=300)$, medicamento $(\mathrm{n}=246)$, pessoas $(\mathrm{n}=185)$, profissionais $(\mathrm{n}=156)$, informações $(\mathrm{n}=142)$, profissional $(\mathrm{n}=138)$, prevenção $(\mathrm{n}=112)$, contatos $(\mathrm{n}=109)$ e família $(\mathrm{n}=109)$ (Figura 1$)$.

\section{Quadro 1}

Questões-chave de referência para as entrevistas semiestruturadas. Alta Floresta, Mato Grosso, Brasil, 2016.

\begin{tabular}{|l|}
\hline 1. As informações relacionadas à PEP estão ajustadas aos valores sociais e conceitos locais (da doença, transmissão, prevenção)? \\
\hline 2. Os grupos-alvo podem explicar as informações que receberam? \\
\hline 3. Qual é a opinião do grupo-alvo sobre a maneira como a informação é fornecida? \\
\hline 4. De acordo com os grupos-alvo, a informação é fornecida em horário e lugar convenientes? \\
\hline 5. Os grupos-alvo se mostraram satisfeitos sobre o modo como os profissionais de saúde se relacionaram com eles? \\
\hline 6. Os grupos-alvo compreendem a percepção da hanseníase e da infecciosidade, uma vez que estão entre o grupo-alvo? \\
\hline
\end{tabular}




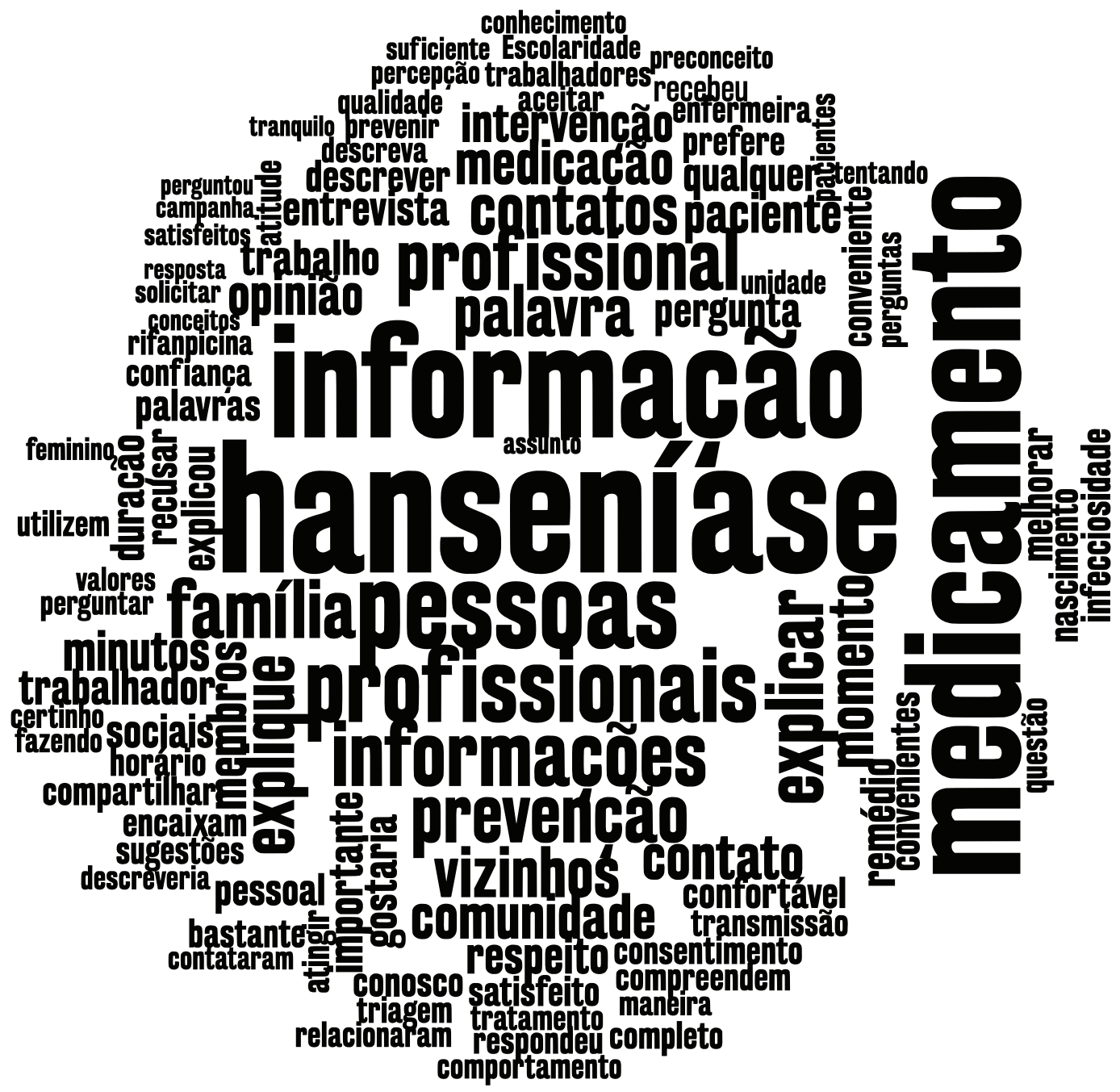

$\mathrm{Na}$ análise do material transcrito, foram identificadas palavras que se repetiam e mensagens que se destacavam nas questões-chave de referência para as entrevistas, relacionadas à oferta da rifampicina como profilaxia da hanseníase. Identificou-se que no Tópico 1 (Quadro 2) os termos "hanseníase”, "lepra" e "MH" (Mycobacterium/Hansen) eram conhecidos entre os entrevistados e apresentavam representações distintas, sendo preterido o termo "hanseníase" entre os grupos-alvos por ser "técnico", "moderno", "leve" e "todos usam", enquanto o termo "lepra” foi recordado como "passado", pelo "isolamento", "preconceito", "sofrimento", "medo" e "ruim".

"...Eu ouvi falar quando eu fui trabalhar na saúde, até então, eu (...) eu num tinha acesso a essa informação de hanseníase, eu ouvia falar de hanseníase, mas eu não sabia que era a lepra antiga (...) do tempo de Cristo..." (PS-10).

"Lá nas histórias da bíblia, falava os leprosos e tal (...), então, a gente já viu na tradução, os leprosos antigos é hoje a hanseníase (...) sempre foi hanseníase, quer dizer, sempre foi a lepra, é a mesma doença (...) só que hoje é um nome mais leve pras pessoas" (V-4). 


\section{Quadro 2}

Relação das palavras ou repetição de ideias mais frequentes encontradas nos tópicos das questões-chave de referência para entrevistas segundo unidade de registro e de contexto. Alta Floresta, Mato Grosso, Brasil, 2016.

\begin{tabular}{|c|c|c|}
\hline Tópicos & Unidade de registo & Unidade de contexto \\
\hline 1 & Hanseníase; Lepra; MH (mycobacterium/Hansen) & $\begin{array}{c}\text { Estigma } \\
\text { Preconceito }\end{array}$ \\
\hline 2 & $\begin{array}{c}\text { O medicamento é para a prevenção; Vizinho; Família; Melhorar; Reforço; Evitar; } \\
\text { Protegido; Imunizar; Combater; Ajudar; Levar saúde; Levando informação; Risco; } \\
\text { Alto contato (muitos casos) }\end{array}$ & $\begin{array}{l}\text { Prevenção } \\
\text { Segurança } \\
\text { Contágio } \\
\text { Transmissão }\end{array}$ \\
\hline 3 & $\begin{array}{c}\text { Não tenho dúvida; Bem informada; Fiquei sabendo; Informação boa; Explicar bem; } \\
\text { Tem paciência; Medicamento entregue em casa; Preocupação com as pessoas; } \\
\text { Tenho medo; Receio; Depende }\end{array}$ & \multirow[t]{3}{*}{ Atitude positiva do profissional de saúde } \\
\hline 4 & $\begin{array}{l}\text { Sim; Horário conveniente; Depende; Reuniu toda a família; } \\
\text { Profissionais marcaram horário }\end{array}$ & \\
\hline 5 & $\begin{array}{l}\text { Bem atendido; Comunicação; Capacitada; Explicar; Orientar; Relação próxima; } \\
\text { Falou certinho; Sempre visitando; Ninguém reclamou }\end{array}$ & \\
\hline 6 & $\begin{array}{c}\text { Medo; Risco da convivência; Reação do medicamento; Falta informação; } \\
\text { Ignorância; Melhor pra mim; Amor à vida; Medicamento é uma prevenção; } \\
\text { É de graça; Melhor prevenir; Evitar o pior }\end{array}$ & $\begin{array}{c}\text { Medo da doença/sequela } \\
\text { Prevenção da doença/sequela } \\
\text { Autocuidado } \\
\text { Acesso }\end{array}$ \\
\hline
\end{tabular}

“...Só ouvia falar na lepra (...) há muitos anos (...) e daí (...) é(...) que é o mesmo né (...) agora tá mais moderno falar hanseniase (...) na nova cultura...” (CS-12).

Tendo em conta as respostas dos grupos-alvo e os termos ou expressões mais frequentes relacionadas ao Tópico 2, verificou-se que as explicações dos entrevistados destacavam aspectos da PEP relacionados à prevenção da hanseníase, à proteção em relação ao contágio e à transmissão. Reconheceram que a família, os vizinhos e a comunidade seriam beneficiados pela estratégia PEP (Quadro 2).

“...Achei que era para a prevenção (...) ela explicou foi porque, como a gente tem vizinhos que tem a doença, $e$ o nosso bairro também tem bastante pessoas que tem a doença, então é (...) o remédio foi pra prevenir, né?” (V-4).

“...Desde que dê certo, é bom, (...) vai ser menos casos” (PS-2).

“...Importância da prevenção, (...) não deixar que a doença avance, e que pegue mais pessoas (...) ela é benção né? É necessária” (CS-3).

Para os Tópicos 3, 4 e 5, referentes à opinião dos grupos-alvo sobre o local, o horário, à orientação sobre a PEP e se ficaram satisfeitos com os profissionais, foi possível identificar que as expressões ou termos relatados se referiam às atitudes positivas dos profissionais de saúde durante a abordagem da PEP (Quadro 2).

“...Sim, até porque elas marcaram né! Elas marcaram para falar com minha tia também, (...) aí elas veio aqui em casa...” (V-5).

“...Daí, (...) ela explicou bem explicado para ele e dai ele tomou” (V-7).

“...Ai varia de família para família. Família que é de 5, de 6 (...) aí o tempo já é mais demorado [referente ao tempo do profissional na casa do entrevistado]” (PS-1).

“...Ela me explicou o que tava acontecendo, o porque deu tomar esse medicamento (...) e aí ela ficou conversando comigo, conversou bastante, tirou minhas dúvidas..." (CS-12).

Quando os entrevistados foram questionados quanto aos motivos para tomar ou não a PEP, reconhecendo estar entre os grupos-alvo (Tópico 6), destacaram-se como unidades de registro o termo "medo" (da doença, de reação ao medicamento ou de sequelas) e a "falta de conhecimento ou informação". Foi possível distinguir atitude de autocuidado pela utilização dos termos "melhor pra mim" 
e "amor à vida" e os termos "prevenção" e "prevenir" foram citados 151 vezes pelos entrevistados (Quadro 2).

Pelas unidades de registro e de contexto, e considerando os sentidos que as palavras assumem quando os grupos-alvos são questionados sobre a oferta da PEP como profilaxia da hanseníase, foi possível distinguir 3 categorias relacionadas à estratégia: Compreensão da PEP, Aceitabilidade e Expectativa da intervenção (Figura 2).

\section{Categoria 1 - Compreensão da PEP}

A compreensão da estratégia PEP foi influenciada pelas atitudes positivas e pelo cuidado das equipes de saúde direcionados às famílias visitadas durante a apresentação da medicação.

Para os indivíduos entrevistados, a rifampicina em dose única é “...pra tentar minimizar os casos da doença...” (CS-12), “...porque a gente vai ter uma forma de prevenir os contatos...” (PS-4). “...É uma prevenção assim, se uma pessoa tomar ali ele fica preparado para não obter a doença (...), a doença nem chegou ainda e as pessoas já estão sendo informadas...” (V-4). “...Porque quando a gente tem muito contato com pessoas que tem [hanseníase] e ainda não começou a tomar o medicamento, a gente pode contrair (...) ser contaminado com essa doença...” (CS-12).

Reconhecem que a estratégia “...é muito boa [PEP] (...) que seria bom se continuasse, (...) pra melhorar a condição de saúde” (PS-9). A medicação, além de impedir a transmissão, é uma estratégia importante “...porque tava prevenindo também as crianças (...) a gente num sabe que pode tá passando para as crianças..." (CI-3).

\section{Figura 2}

Fluxograma representativo das categorias de análise relacionadas à PEP. Alta Floresta, Mato Grosso, Brasil, 2016.

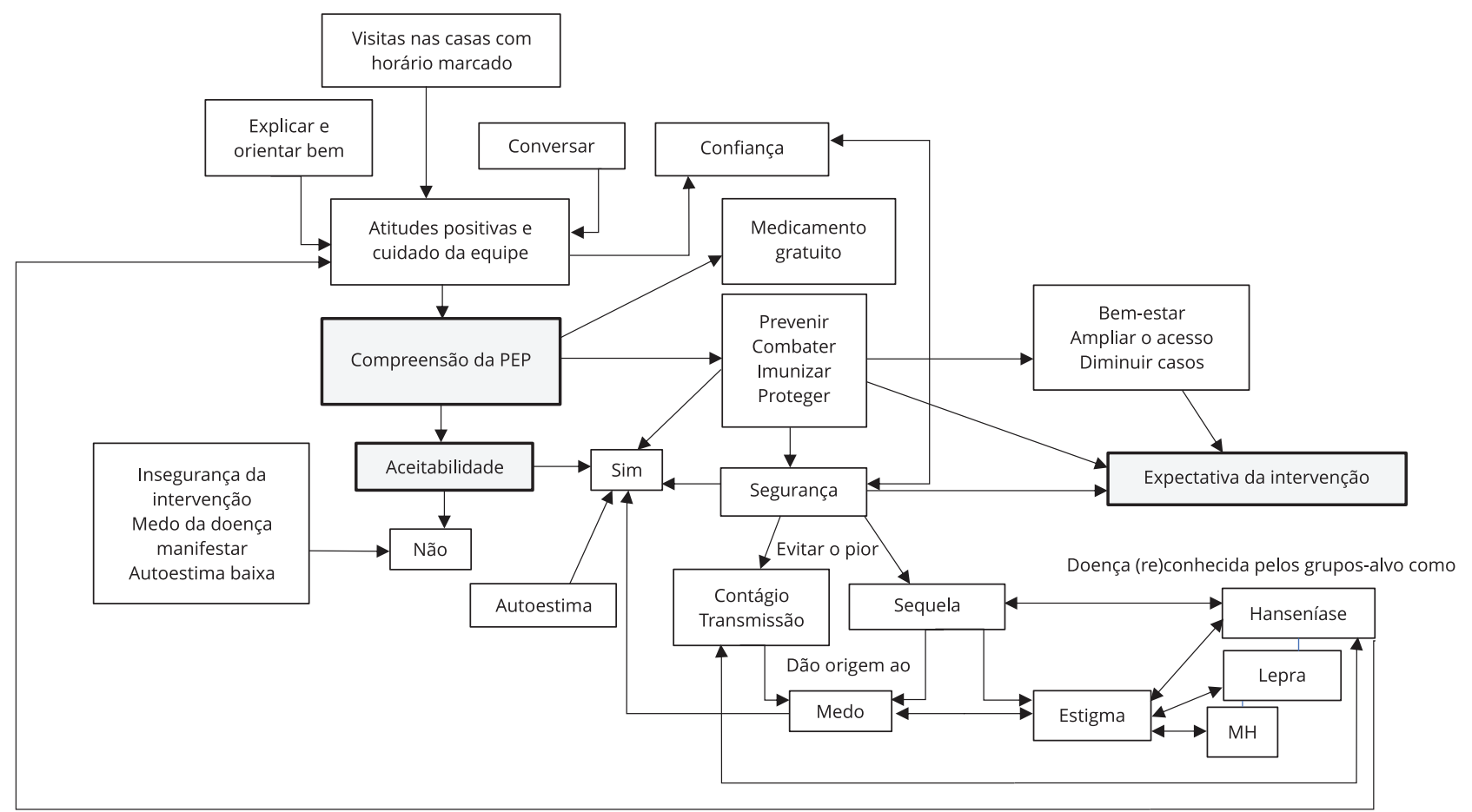




\section{Categoria 2 - Aceitabilidade}

Ainda que a compreensão da PEP como medida de prevenção e proteção tenha contribuído para os entrevistados aceitarem e tomarem a medicação, a confiança e a segurança nos profissionais parecem ter influenciado na decisão tomada.

Dentre os motivos relacionados à confiança e segurança, estão as relações estabelecidas entre as equipes de saúde e as famílias na comunidade, “...porque a gente se conhece (...) o bairro é pequeno, então acabo confiando bastante...” (V-8). Acrescenta-se, receber informação sobre o que será realizado no bairro “...porque o que a gente vai tomar, a gente fica sabendo sobre o que é..." (V-8). Outro aspecto relacionado foi “... a identificação do profissional como saúde, a pessoa já olha diferente...” (CS-11).

Acrescenta-se à aceitabilidade, o fato de reconhecerem que a rifampicina poderá evitar o pior, ou seja, a ocorrência de sequelas e o contágio. Nas falas transcritas, as sequelas e a possibilidade de contágio apresentaram-se como geradores do medo, evidenciado o preconceito e o estigma, “...porque não é fácil (...) só quem passa sabe, porque mexeu muito comigo. A minha família passou muito difícil...” (CS-6), “...tem preconceito, esse negócio tem história triste [referindo-se à lepra]” (V-1). “...Ela é contagiosa né (...) Ela transmite..." (V-10).

\section{Categoria 3 - Expectativa da intervenção}

Os entrevistados esperam que a estratégia com rifampicina possa "...diminuir o número de pessoas com hanseníase, né!" (V-4), “...porque se esse medicamento fazer esse bloqueio (...), vai ser muito bom...” (PS-3), “... pode melhorar porque vai reduzir o risco de hanseníase...” (PS-1).

Identificou-se relacionado à expectativa da intervenção um sentimento de alívio, de bem-estar, não somente pela proteção atribuída à rifampicina, mas também pelo fato de esta medicação ser distribuída na comunidade. "Sinto aliviado porque elas saem de porta em porta ajudando todo mundo, (...) sinto mais protegida depois de tomar o remédio..." (V-6). "...Porque se você está prevenindo uma coisa que você não tem, é bom pra todo mundo..." (V-2).

\section{Discussão}

A aceitabilidade da quimioprofilaxia com rifampicina em dose única entre os grupos-alvo mostrou-se ligada à compreensão dos entrevistados de que a medicação poderia proteger, prevenir a ocorrência de casos novos e evitar a transmissão. Foi observado que a autoestima, a segurança e a confiança nos profissionais de saúde influenciaram positivamente os resultados alcançados.

Estudos com abordagem sobre a compreensão de uma implementação bem-sucedida são escassos na literatura e envolvem conceitos que, segundo Proctor et al. 11, estão relacionados aos resultados da implementação (aceitabilidade, adoção, custo, viabilidade, sustentabilidade); aos resultados do serviço (eficiência, eficácia, segurança, centralidade no paciente) e do paciente (satisfação, sintomatologia).

A aceitabilidade está entre os conceitos-chaves de linguagem e conceituação relacionados à compreensão e ao entendimento de uma implementação em saúde. Representa a percepção de que um serviço, tratamento ou inovação é bom ou considerado apropriado 11,16,17, como pode ser observado na fala dos entrevistados.

A PEP é compreendida como estratégia preventiva, uma vez que "a doença nem chegou" e "ele fica preparado para não obter a doença". Ela favorecerá o controle ou diminuição da doença, pois poderá "diminuir os casos". A percepção de que a PEP é apropriada também se mostrou relacionada à interrupção da transmissão da doença e por "melhorar a condição de saúde".

A compreensão da estratégia com rifampicina em dose única para quimioprofilaxia da hanseníase entre os grupos-alvo mostrou-se influenciada pelos cuidados das equipes de saúde no que tange à operacionalização das ações e à maneira como as relações interpessoais foram estabelecidas. Tais cuidados foram expressos quando "ela explicou bem explicado", quando o "médico passa a instrução pra ela e elas sai de porta em porta explicando como é", porque "as informação delas foi boa e elas trataram super bem" (CI-6) e se "a pessoa começou a fazer uma queixa eu já fico, a gente fica atento... já ligo os fatos pra ver se é(...) e ajudar mais rápido" (PS-3). Acrescenta-se o agendamento das visitas nas casas em tempo oportuno 
para os moradores e com priorização de todos os membros da família durante as explicações da PEP. Quando necessário, os indivíduos foram visitados no final de semana ou no período noturno.

Em razão de a própria equipe de saúde do bairro ser a responsável pela entrega da medicação, os entrevistados sentiram-se mais à vontade e seguros para agendar um horário mais adequado, o que demonstra o papel da atenção primária nas ações de vigilância como a quimioprofilaxia.

A organização das visitas domiciliares para as informações e oferta da PEP com mobilização familiar aponta a família como objeto do cuidado e a valorização das particularidades de cada núcleo residencial. Essas ações são congruentes ao modelo de atenção à saúde estabelecido pela implantação da Estratégia Saúde da Família em que o foco de atenção passou ser a família e seu ambiente de vida. Nesse espaço, onde se constroem as relações intra e extrafamiliares, é possível uma compreensão ampliada do processo saúde-doença e da necessidade de intervenções de maior impacto e significação social, direcionadas à vigilância em saúde 18 .

Sabe-se que as condições de saúde-doença dos membros da família e a família como unidade se influenciam mutuamente, e o cuidado centrado no paciente permite o conhecimento das preferências, dos valores e das necessidades individuais, condição relevante para nortear as práticas em saúde 19,20.

No respeitante ao diagnóstico de hanseníase em algum membro da família, foi possível perceber, entre os grupos-alvo entrevistados, a influência da doença no contexto familiar e nas relações sociais em razão de a percepção da doença permanecer relacionada às memórias e crenças do passado, ao estigma.

As atitudes positivas e o cuidado dos profissionais durante a oferta da PEP contribuíram para o sentimento de confiança e segurança dos grupos-alvo, favorecendo a aceitabilidade da medicação como um resultado positivo da implementação, porque "você chegar de surpresa na casa da pessoa e chegar e falar para ele eu vim te dar esse remédio, (...) eu acho que ele leva um choque maior" (PS-2). Em concordância com Silva et al. 19, destaca-se o potencial das equipes de saúde para desenvolverem o trabalho com famílias, a criação de vínculos e o estabelecimento de relações mais humanas.

Durante o período de implantação da oferta de rifampicina para quimioprofilaxia da hanseníase, ocorreram reuniões e capacitações das equipes envolvidas na estratégia. Buscou-se, na operacionalização das ações, organizar as atividades de maneira que um maior número de pessoas tivesse a oportunidade de receber as explicações e informações relacionadas à PEP. A maneira como os profissionais de saúde receberam e passaram as informações sobre a PEP, possivelmente influenciou na aceitabilidade das pessoas envolvidas, o que inclui também os profissionais 21 . Acrescenta-se que a confiança gerada nos grupos-alvos foi importante para garantir a segurança em relação à estratégia e credibilidade no profissional.

O resultado de uma ação coletiva na comunidade, como a implementação de um programa pela introdução de uma nova tecnologia em saúde, demanda o envolvimento de todos aqueles envolvidos no processo e representa uma avaliação subjetiva feita por indivíduos que experimentam (ou esperam experimentar) ou realizam (ou esperam realizar) uma intervenção.

Encontrou-se aceitação pelos grupos entrevistados por referirem que a estratégia ou programa foi reconhecido como "muito bom" e "...deveria continuar se mostrasse resultados" (PS-9), "...porque prevenir é a melhor coisa que tem..." e "é de graça" (CI-5). Também se verificou possível impacto na "visão" sobre a hanseníase, já que “...se tiver um remédio pra evitar passar pro resto da família, a pessoa fica mais à vontade”, "eu fico feliz" (CTI-4), contudo a ocorrência de efeitos colaterais devido à rifampicina foi pontuada como uma expressão condicionada à aceitabilidade, considerando que, “...mas se não sentir mal, acho que é bom”.

Como descrito, os grupos se mostraram satisfeitos com a nova intervenção, reconhecendo a estratégia como uma oportunidade para prevenção de contatos e controle da doença nos lugares de maior risco, "por causa do alto contato que tem por perto, (...) então a gente estamos em área de risco, por que hanseníase é transmitido, né?” (PS-1). Também demonstraram sentimento de mais tranquilidade quanto a ficarem protegidos, "porque se não tivesse eu ia ter que correr atrás de um remédio pra eu e meus filhos tomar, pra não pegar a doença, (...) porque eu ficava com medo de pegar em mim quando ele [o marido] deitava perto de mim" (CTI-4). Ratificando a influência do processo saúde-doença nas relações familiares, para CTI-4, o convívio com alguém doente gerou medo e insegurança.

Identificou-se também contraposição de alguns entrevistados quanto à estratégia PEP e seus resultados. Tal fato confirma a necessidade de mais esclarecimentos e investimentos na capacitação dos profissionais para a apresentação da estratégia aos grupos-alvo, visto que a confiança dos entre- 
vistados nos profissionais de saúde se mostrou relacionada a aceitar a rifampicina. Ressalta-se que este estudo foi realizado nos meses iniciais do projeto PEP. No entanto, sabendo-se que a rotatividade de profissionais na atenção primária acontece em todo o país, os esclarecimentos e capacitações para o protocolo da PEP de hanseníase será uma demanda em caso de utilização na rotina dos serviços de saúde.

Frisa-se que um termo utilizado somente entre os profissionais de saúde foi "MH". Entre os profissionais, é um termo "prático" e "mais fácil de comunicar". Representa uma maneira de "preservar" ou evitar exposição do paciente na unidade de saúde. Porém, ainda que essa maneira de "preservar" a identidade do paciente represente uma abordagem ética, também revela uma restrição ao termo "hanseníase", sobretudo quando o profissional o utiliza fora da unidade de saúde.

Observou-se que o termo "lepra" foi associado à doença de pele, às crenças e ao passado, quando os indivíduos eram isolados e separados do restante da comunidade, enquanto o termo "hanseníase" relacionou-se à doença que compromete o nervo e, ao mesmo tempo, tem cura. No entanto, a doença (lepra ou hanseníase) mantém de forma persistente o preconceito.

Indivíduos que passaram por mudanças significativas no seu dia a dia, após apresentarem a doença, trazem experiências que precisam ser valorizadas durante um diálogo, como o relato apresentado por CI-8, pois podem manter o preconceito e estigma da doença.

"Só sentia ela [hanseníase], a borbulharada, acabou o homem. Eu fiquei na minha, porque se tiver que matar mata, tiver de sarar, sara. Eu sou animado, não tem tristeza não". "Ele mata a hanseníase [a medicação], mas a saúde não volta mais. Vai ser a mesma coisa" (CI-8).

No referente às medidas de prevenção e à promoção da saúde, espera-se que a capacitação profissional contribua para o fortalecimento e a efetivação de práticas porque envolve o aprimoramento e implementação de técnicas. Todavia, percebe-se na fala de um profissional de saúde a necessidade além da técnica. Ele enfatiza que, "mesmo sendo uma coisa nova e criando novas expectativas", sua "visão" sobre a hanseníase não mudou. Novamente, é possível constatar que a doença traz representações sociais ancoradas no termo "lepra", situação que pode fortalecer o estigma entre os profissionais de saúde com reflexo na comunidade 22.

Ainda que o estigma não tenha sido objeto deste estudo, quando presente entre os profissionais de saúde como condição indutora de autopercepções negativas, desvantagens sociais ou de exclusão, ele pode ser considerado como um fator iatrogênico e, possivelmente, poderia comprometer a viabilização da PEP 23,24.

A maioria dos profissionais que ofereceu a medicação sentiu-se útil em poder colaborar para a "prevenção" da hanseníase, "prevenir os contatos", para "melhorar a condição de saúde" uma vez que "senti que estava ajudando, que ia fazer bem a eles [contatos]". As atividades realizadas pelos profissionais proporcionaram satisfação, felicidade, gratificação embora apresentassem inseguranças quanto à estratégia em implantação.

Ter comorbidade foi uma das causas responsáveis pela impossibilidade de tomar rifampicina, assim como considerar a "medicação forte" $(\mathrm{V}-12)$ e "ter medo de tomar a medicação e a doença se manifestar" (CS-20). Para os entrevistados, a falta de informação ou informação inadequada poderá levar os indivíduos a rejeitarem a rifampicina.

A expectativa de prevenção e proteção quanto à manifestação da hanseníase, assim como a possibilidade de controle da evolução da doença para as formas mais graves foram algumas afirmativas relacionadas à razão de se aceitar e tomar a PEP.

Espera-se que as novas tecnologias possam impactar não somente no controle da hanseníase, mas também contribuir para o resgate da cidadania e do respeito pelas pessoas afetadas pela doença.

\section{Considerações finais}

A estratégia com rifampicina em dose única para profilaxia da hanseníase se apresentou aceitável e com potencial impacto na redução do estigma da doença pelo envolvimento de todos os profissionais de saúde para sua implementação. As visitas em domicílio e a abordagem da PEP na oferta da medicação aproximaram profissionais e comunidade, influenciando as relações inter e extrafamiliares pelo 
fortalecimento de vínculos relacionados à confiança, segurança e expectativas futuras de proteção, prevenção e bem-estar.

Houve reconhecimento da relevância da estratégia PEP pela possibilidade de interrupção da cadeia de transmissão, diminuição de casos novos e melhora na qualidade de vida. Entretanto, o medo da doença e o estigma ainda perceptível nas falas dos grupos-alvo apontam a necessidade de capacitação contínua das equipes de saúde com enfoque na percepção da doença, dos valores familiares e de suas experiências com a hanseníase.

O grau de parentesco e a compreensão sobre a doença não se mostraram suficientes para influenciar a aceitabilidade do medicamento. A insegurança em tomar a medicação e de a doença se manifestar influenciaram negativamente a aceitação da PEP; por outro lado, as informações prévias sobre a estratégia PEP contribuíram para o fortalecimento da confiança nos profissionais de saúde e para a aceitabilidade da medicação.

\section{Colaboradores}

D. C. B. Cortela participou durante a coleta de dados, análise e interpretação dos dados, redação do artigo e aprovação final da versão a ser publicada. S. M. B. Ferreira contribuiu durante a coleta de dados, interpretação dos dados, revisão crítica relevante do conteúdo intelectual e aprovação final da versão a ser publicada. M. C. L. Virmond, L. Mieras, P. Steinmann e A. Cavaliero participaram da concepção e projeto, revisão crítica relevante do conteúdo intelectual e aprovação final da versão a ser publicada. E. Ignotti participou durante a concepção e projeto, análise e interpretação dos dados, revisão crítica relevante do conteúdo intelectual e aprovação final da versão a ser publicada.

\section{Informações adicionais}

ORCID: Denise da Costa Boamorte Cortela (00000003-0052-6216); Silvana Margarida Benevidez Ferreira (0000-0001-7421-5803); Marcos Cunha Lopes Virmond (0000-0002-1395-639X); Liesbeth Mieras (0000-0001-6943-1712); Peter Steinmann (0000-0003-4800-3019); Eliane Ignotti (00000002-9743-1856); Arielle Cavaliero (0000-00017064-6403).

\section{Conflito de interesses}

A coautora A. Cavaliero é profissional técnica da Fundação Novartis.

\section{Agradecimentos}

À Fundação Uniselva (UFMT) e Fundação Novartis pelo auxílio financeiro.

\section{Referências}

1. Cortela DCB, Ignotti E. Conhecimento e experiências do cirurgião-dentista sobre hanseníase em Cáceres, MT, Brasil. Rev Odonto Ciênc 2008; 23:243-50.

2. Santos AS, Castro DS, Falqueto A. Fatores de risco para transmissão da hanseníase. Rev Bras Enferm 2008; 61:738-43.

3. Freitas BHBM, Xavier DR, Cortela DCB, Ferreira SMB. Hanseníase em menores de quinze anos em municípios prioritários, Mato Grosso, Brasil. Rev Bras Epidemiol 2018; 21:e180016.

4. Marciano LHSC, Belone AFF, Rosa PS, Coelho NMB, Ghidella CC, Nardi SMT, et al. Epidemiological and geographical characterization of leprosy in a Brazilian hyperendemic municipality. Cad Saúde Pública 2018; 34:e00197216.

5. Smith CM, Smith WCS. Chemoprophylaxis is effective in the prevention of leprosy in endemic countries: a systematic review and meta-analysis. J Infect 2000; 41:137-42.

6. Comissão Nacional de Incorporação de Tecnologias no SUS. Quimioprofilaxia de contatos de doentes de hanseníase com rifampicina em dose única. Brasília: Secretaria de Ciência, Tecnologia e Insumos Estratégicos, Ministério da Saúde; 2015. (Relatório de Recomendação, 165).

7. Moet FJ, Pahan D, Oskam L, Richardus JH; COLEP Study Group. Effectiveness of single dose rifampicin in preventing leprosy in close contacts of patients with newly diagnosed leprosy: cluster randomised controlled trial. BMJ 2008; 336:761-4.

8. Richardus J, Oskam L. Protecting people against leprosy: chemoprophylaxis and immunoprophylaxis Clin Dermatol 2015; 33:19-25.

9. Ferreira SMB, Yonekura T, Ignotti E, Oliveira LB, Takahashi J, Soares CB. Effectiveness of rifampicin chemoprophylaxis in preventing leprosy in patient contacts: a systematic review of quantitative and qualitative evidence. JBI Database System Rev Implement Rep 2017; 15:2555-84. 
10. Schutten K. Community perception of leprosy: baseline assessment of perceptions - knowledge, beliefs, attitudes, and behaviour in EastJava, Indonesia [PhD Thesis]. Amsterdam: Vrije Universiteit Amsterdam; 2018.

11. Proctor E, Silmere H, Raghavan R, Hovmand P, Aarons G, Bunger A, et al. Outcomes for implementation research: conceptual distinctions, measurement challenges, and research agenda. Adm Policy Ment Health 2011; 38:6576.

12. Becker MH, Maiman LA. Sociobehavioral determinants of compliance with health and medical care recommendations. Med Care $1975 ; 13: 10-24$.

13. Gomez PF, Gutiérrez MGR, Moreira RSL. Percepção da doença: uma avaliação a ser realizada pelos enfermeiros. Rev Bras Enferm 2011; 64:925-30.

14. Barth-Jaeggi T, Steinmann P, Mieras L, van Brakel W, Richardus JH, Tiwari A, et al. Leprosy Post-Exposure Prophylaxis (LPEP) programme: study protocol for evaluating the feasibility and impact on case detection rates of contact tracing and single dose rifampicin. BMJ Open 2016; 6:e013633.

15. Bardin L. Análise de conteúdo. Lisboa: Edições 70; 1977.

16. Proctor EK, Landsverk J, Aarons G, Chambers D, Glisson C, Mittman B. Implementation research in mental health services: an emerging science with conceptual, methodological, and training challenges. Adm Policy Ment Health 2009; 36:24-34.
17. Sekhon M, Cartwright M, Francis JJ. Acceptability of healthcare interventions: an overview of reviews and development of a theoretical framework. BMC Health Serv Res 2017; $17: 88$.

18. Coordenação de Saúde da Comunidade, Secretaria de Assistência à Saúde, Ministério da Saúde. Saúde da família: uma estratégia para a reorientação do modelo assistencial. Brasília: Ministério da Saúde; 1997.

19. Silva MCLSR, Silva L, Bousso RS. A abordagem à família na Estratégia Saúde da Família: uma revisão integrativa da literatura. Rev Esc Enferm USP 2011; 45:1250-5.

20. Donabedian A. The quality of medical care. Science 1978; 200:856-64.

21. Sidani S, Epstein DR, Bootzin RR, Moritz P, Miranda J. Assessment of preferences for treatment: validation of a measure. Res Nurs Health 2009; 32:419-31.

22. Oliveira M, Mendes C, Tardin RT, Cunha M, Arruda A. Social representation of Hansen's disease thirty years after the term "leprosy" was replaced in Brazil. Hist Ciênc Saúde-Manguinhos 2003; 10 Suppl 1:41-8.

23. White C. Iatrogenic stigma in outpatient treatment for Hansen's disease (leprosy) in Brazil. Health Educ Res 2008; 23:25-39.

24. Nations MK, Lira GV, Catrib AMF. Stigma, deforming metaphors and patients' moral experience of multibacillary leprosy in Sobral, Ceará State, Brazil. Cad Saúde Pública 2009; 25:1215-24 


\section{Abstract}

The aim was to analyze the acceptability of chemoprophylaxis with single-dose rifampicin (PEP) in contacts, index leprosy cases, and health professionals and related factors that can influence adherence. A qualitative content analysis study was performed after application of semi-structured interviews according to the protocol proposed in the LPEP program (2016) drafted at Alta Floresta, Mato Grosso State, Brazil, in July 2016. Study participants included individuals with leprosy, contacts, and health professionals. The QRS NVivo software version 10 was used. A total of 80 individuals were contacted, including 54 (67\%) contacts, $11(14 \%)$ index cases, and 15 (19\%) health professionals. $94 \%$ of the contacts $(51 / 54)$ took PEP. Three PEP categories were identified: understanding, acceptance, and expectation towards the intervention. Understanding proved to be related to care by the health team. Acceptance (or lack thereof) of the medication was related to fear, trust, and protection, the strategy's operability, self-esteem, and insecurity regarding the intervention. Expectation towards the intervention was related to wellbeing, prevention of the disease, sequelae, decrease in public expenditures, and expanded access. Participants acknowledged the relevance of the PEP strategy based on the possibility of interrupting the transmission chain, reduction in new cases, and improved quality of life. Insecurity in taking the medication and the possibility of the disease manifesting itself had a negative influence on acceptance of PEP, while prior information on the PEP strategy helped strengthen trust in the health professionals and the medication's acceptance.

Rifampicin; Chemoprophylaxis; Health Knowledge, Attitudes, Practice; Patient Acceptance of Health Care

\section{Resumen}

El objetivo fue analizar la aceptabilidad de la quimioprofilaxis con rifampicina en dosis única (PEP) entre los contactos, casos indices de hanseniasis y profesionales de salud, así como los factores relacionados que puedan influenciar en la adhesión al tratamiento. Se realizó un estudio cualitativo de análisis de contenido, tras la realización de entrevistas semiestructuradas, según el protocolo propuesto en el programa LPEP (2016), realizado en Alta Floresta, Mato Grosso, Brasil, en julio de 2016. Participaron en el estudio individuos diagnosticados con hanseniasis, contactos y profesionales de la salud. Se utilizó el software QRS NVivo versión 10. Se contactó con 80 individuos, siendo 54 (67\%) contactos, 11 (14\%) casos indices y 15 (19\%) profesionales de salud. Entre los contactos 94\% (51/54) tomaron PEP. Se identificaron 3 categorías respecto a la PEP: comprensión, aceptación y expectativa de intervención. La comprensión estuvo relacionada con el cuidado del equipo de salud. El aceptar o no la medicación estuvo relacionado con el miedo, confianza y protección, operatividad de la estrategia, autoestima e inseguridad de la intervención. La expectativa de la intervención estuvo relacionada con el bienestar, prevención de la enfermedad, así como secuelas, disminución de gasto público y ampliación del acceso. Existió un reconocimiento de la relevancia de la estrategia PEP por la posibilidad de interrupción de la cadena de transmisión, disminución de casos nuevos y mejora en la calidad de vida. La inseguridad en tomar la medicación y de que la enfermedad se manifestara influenciaron negativamente en la aceptación de la PEP, por otro lado, la información previa sobre la estrategia PEP contribuyó al fortalecimiento de la confianza en los profesionales de salud y a la aceptabilidad de la medicación.

Rifampicina; Quimioprofilaxis; Conocimientos, Actitudes y Práctica en Salud; Aceptación de la Atención de Salud
Recebido em 19/Abr/2019

Versão final reapresentada em 24/Jul/2019

Aprovado em 03/Set/2019 